\title{
MICROMECHANICAL FORCE SENSING WITH APPLICATIONS IN BIOLOGY
}

\author{
Thomas W. Kenny, Yiching Liang, Su-Wen Ueng, Michael Bartsch and Robert Rudnitsky
} Departments of Mechanical Engineering and Applied Physics, Stanford University,
Stanford, CA 94305, Kenny@mems.stanford.edu

\begin{abstract}
In recent years, MEMS devices have been developed for a wide variety of applications. Many of the signals that these sensors are intended to detect are expressed as forces that stress or deflect the micromechanical structure. As sensors are miniaturized, these forces naturally become smaller, and techniques for detection are required to improve. As the force sensing capabilities of MEMS devices have improved, it has become possible to apply these devices to interesting scientific experiments on biological systems.
\end{abstract}

\section{INTRODUCTION}

Since the 1970s, silicon fabrication tools have been adapted and extended to allow fabrication of micromechanical structures [1]. In recent years, this field has grown to include a bewildering variety of resonators, pumps, gear trains, inertial sensors, optical switches, and many other devices. And, the fabrication tool set has expanded to include use of thick photoresists, high aspect ratio plasma etching, and materials such as acrylic. The expansion of the tools and applications can be expected to continue as interest in bioanalytical systems, and telecommunications applications grows. Sensors make up a significant subset of all micromechanical devices. Most of these devices operate by sensing forces derived from the signal of interest. As examples, pressure sensors detect forces on diaphragms, inertial sensors detect forces on test masses, magnetometers detect torque on magnets, and so on.

Before the development of micromechanical devices, macroscopic force sensors were used in many instruments. Bonded strain gauges, optical deflectors, differential capacitors, and other instrumental means were used to convert macroscopic physical signals into electrical signals. MEMS devices enabled a completely new approach to many common instruments, and the opportunity for miniaturization of these instruments enables force sensing to become important in many new applications. In general, miniaturization of the dimensions of a force sensor imposes a reduction in the force to be detected. For example, the force available for a pressure sensor is necessarily proportional to the area of the diaphragm. The force available for inertial sensors generally scales as the mass, which scales as the cube of the dimension. Therefore, miniature sensors are generally required to detect smaller forces than macrosensors.

Using micromechanical structures and integrated sensors, it is possible to detect forces over a very wide range. In this paper, the sizes of the forces that are detected in a variety of existing micrometer and nanometer scale devices will be outlined. It will be seen that forces ranging from milli-Newtons to atto-Newtons are detectable using appropriately-designed devices. This paper will discuss work in this area and opportunities for important measurements and applications, particularly in the area of biological science.

\section{FORCE-SENSING IN PIEZORESISTIVE MEMS DEVICES}

Before discussing custom-force sensors, some examples of force sensing in commercial MEMS devices will be presented. This discussion will illustrate some of the basic methods for force sensing, and the very impressive performance achieved with "conventional" low-cost MEMS devices.

The first example is a standard piezoresistive pressure sensor, such as the Lucas Novasensor NP301 [2]. This sensor is fabricated by standard etching of silicon, wafer bonding (to form a sealed vacuum cavity), and ion implantation (to form piezoresistive strain gauges on the diaphragm). These devices are manufactured in quantities of millions annually, sold for a few dollars (plus packaging costs), and are a well-established example of simple, but profitable MEMS devices.

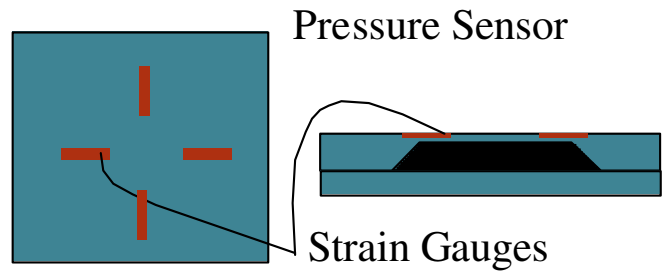

Figure 1 Drawing of a Piezoresistive Pressure Sensor, such as the Lucas Novasensor NP301. The piezoresistive strain gauges act as resistors whose resistance is a function of the pressureinduced strain in the thin silicon diaphragm. An off-chip wheatstone bridge readout circuit allows straightforward measurement of the pressure difference between the outside and the sealed reference cavity.

This particular micromachined sensor has a diaphragm area of about $(1 \mathrm{~mm})^{2}$, and can detect pressures up to 15 PSI. This corresponds to a maximum force of $25 \mathrm{mN}$. These devices are specified to an accuracy of $0.5 \%$ of the full-scale signal, corresponding to a force accuracy of $100 \mu \mathrm{N}$. Individual devices may be calibrated to an accuracy of about 20-50x better then the lot specification, so individual $\mathrm{mm}$-scale micromachined pressure sensors are capable of force detection from $25 \mathrm{mN}$ down to $5 \mu \mathrm{N}$. If the noise of a pressure sensor is measured with a simple, off-chip instrumentation amplifier, the voltage fluctuations correspond to forces as small as $10 \mathrm{nN}$ [3].

This is a very impressive performance capability. For contrast, the standard instrument for measuring small forces in one axis is the conventional load cell. There are many commercial suppliers of load cells, some of whom specialize in load cells for relatively small forces. In order to measure down to $1 \mathrm{mN}$ with a commercial load cell, it is necessary to spend more than $\$ 400$, and the purchased devices suffer from drift so that offset adjustments are constantly required. The piezoresistive pressure sensor is more than 100x more accurate for small forces, and is available at about 100x less cost. 
Piezoresistive sensors have been used for many other MEMS applications, including accelerometers [4,5], gyroscopes [6], and AFM cantilevers [7]. The primary advantage of this approach is that the sensor impedance is relatively low (a few $\mathrm{K} \Omega$ ), and it is possible to extract small signals without interference from noise with off-chip integrated circuits. Therefore, low-cost, two-chip hybrid systems can be built without the need to develop an integrated MEMS/Electronics process.

There has been recent interest in the development of ultrathin piezoresistive cantilevers for various applications. The main reason for this approach is that the sensitivity should improve dramatically as the thickness is reduced. However, it is also necessary to confine the piezoresistor into a fraction of the beam thickness, and this becomes a challenge for beam thickness less than $2 \mu \mathrm{m}$. Growth of very thin films of doped epitaxial silicon has allowed development of piezoresistive cantilevers thinner than $0.1 \mu \mathrm{m}$, and these cantilevers have been shown by Harley and Liang to be capable of detecting forces as small as $1 \mathrm{fN}$. [8,9]

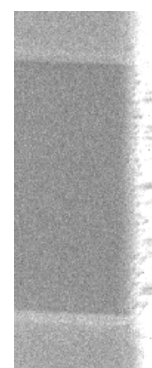

Figure 2 cantilever. This cantilever is $70 \mathrm{~nm}$ thick, and includes a $20 \mathrm{~nm}$ n-type piezoresistor. This cantilever has force resolution of 1.6 $\mathrm{fN} / \mathrm{Hz}$ at $1 \mathrm{kHz}$ at room temperature. [9]

\section{FORCE SENSING IN A CAPACITIVE ACCELEROMETER}

Our second micrometer-scale MEMS example is a surface micromachined accelerometer, the ADXL202 [10]. This device is a member of the Analog ADXL family, in which $2 \mu \mathrm{m}$-thick polysilicon masses are suspended above a surface in the middle of an integrated circuit. The deflection of this small proof mass is detected by differential capacitance measurement between arrays of fingers on the edge of the mass and mounted on the surrounding substrate. Because of the small total capacitance involved $(<0.2 \mathrm{pF})$, an integrated circuit with a preamplifier positioned as close as possible to the capacitor is required.

The micromechanical structure consists of a layer of polysilicon with thickness of $2 \mu \mathrm{m}$, mass of $10^{-10} \mathrm{~kg}$, suspension stiffness of about $1 \mathrm{~N} / \mathrm{m}$. The minimum detectable acceleration (in a $1 \mathrm{~Hz}$ bandwidth) is approximately $0.5 \mathrm{mg}$ (where $1 \mathrm{~g}=9.8 \mathrm{~m} / \mathrm{s}^{2}$ ). For this device structure, this acceleration corresponds to a displacement of about 0.005 Angstroms $(0.5 \mathrm{pm})$, and results in a force on the proof mass of about $0.5 \mathrm{pN}$. This is a phenomenal performance level for an off-the-shelf component that is widely sold for as little as $\$ 10$. This performance level is very close to thermodynamic limits for force detection, and represents a very impressive accomplishment within a large scale production environment.
There are various noise sources to be concerned with in miniature accelerometers, many of which will impose some limit on the minimum detectable signal. At a fundamental level, there is a thermomechanical noise mechanism to be concerned with. This mechanism is the mechanical analog of Johnson Noise in resistors, in which energy dissipation necessarily introduces fluctuations through thermodynamics. For the case of accelerometers, this fluctuation may be represented as a 'Noise force' applied to the proof mass. This noise source has been analyzed for accelerometers [11], and results in a minimum detectable acceleration of

$$
A_{\text {min }}=\sqrt{\frac{4 k_{B} \omega_{o} \Delta F}{m Q}},
$$

where $k_{B}$ is Boltzmann's constant, $\square_{\mathrm{o}}$ is the resonant frequency of the suspension for the proof mass, $\mathrm{m}$ is the mass value, and $\mathrm{Q}$ is the Quality Factor of the resonance (approximately equal to the number of free oscillations that will occur before damping causes the oscillation amplitude to decay by 1/e). This thermodynamic limit cannot be avoided, and this limit becomes significant where $\mathrm{m}$ becomes very small.

It is possible to work around this issue through the use of feedback control. In most cases, the sensitivity (V/g) of an accelerometer is proportional to $1 / \omega_{0}{ }^{2}$, so it is useful to select a low value of resonant frequency. In addition, this helps reduce the thermomechanical noise. However, the resonance frequency also determines the bandwidth of the accelerometer. Feedback control can be used to modify the apparent dynamics of the device by imposing force feedback to "stiffen" the suspension. For an accelerometer, this can allow operation above the openloop resonance without the responsivity and noise issues that would accompany an increased resonance. This method is used in conventional seismometers [12] and conventional inertial navigation accelerometers [13], and has been adapted to a MEMS accelerometer with a tunneling transducer [14].

In the ADXL accelerometer example discussed here, the minimum detectable acceleration (assuming $\mathrm{Q}=0.7$ ) is $0.3 \mathrm{mg}$ in a $1-\mathrm{Hz}$ bandwidth. This is less than a factor of two smaller than the specified performance for these accelerometers, and represents a final limit to the performance of accelerometers based on a surface-micromachined architecture. Nevertheless, this MEMS device is operating by detecting forces smaller than $1 \mathrm{pN}$, reflecting what is possible with highly-integrated differential capacitive sensing.

\section{FORCE SENSING IN GYROSCOPES}

There has been significant recent interest in the use of gyroscopes to detect errors in automobile orientation, and to trigger corrective differential braking to achieve active skid control. Bosch manufactures a miniature Coriolis Force gyroscope for this application, and it represents another impressive example of the detection of small forces.

In this device, a proof mass of less than $0.01 \mathrm{mg}$ is excited into resonant oscillation at frequency of $500 \mathrm{~Hz}$ with amplitude of approximately $10 \mu \mathrm{m}$. Rotations of the sensor about an axis perpendicular to the motion of the mass cause an oscillating Coriolis force with amplitude :

$$
F_{\text {Coriolis }}=m \omega x V,
$$

where " $\mathrm{x}$ " is the vector cross-product between the rotation vector and the velocity vector. The velocity of the mass is 
oscillating with amplitude equal to the product of the amplitude and oscillation frequency. For a rotation of 1 degree/sec, this oscillating Coriolis force has amplitude of $6 \mathrm{pN}$. One advantage for detection of this force is that it appears as an oscillating signal, rather than as a DC signal. Unfortunately, there are many error sources to be overcome in a Coriolis gyro, including cross-coupling between the lateral modes, and thermallyenhanced cross-coupling. Thermal noise also imposes a fundamental limit for force detection in a gyroscope [15].

$$
\Omega_{\text {min }}=\frac{1}{4 C_{g} \text { Amplitude }} \sqrt{\frac{4 k_{B} T}{\omega_{o} m Q}}
$$

For the parameters described above, thermal noise limits detection to signals greater than 0.1 degree/sec in a $1 \mathrm{~Hz}$ bandwidth. These issues were discussed at greater length by Geen at Hilton Head '98 [16].

\section{FORCE SENSING IN OTHER MEMS DEVICES}

In addition to piezoresistive sensing and capacitive sensing, it is possible to use optical transducers. AFM cantilever deflection is typically sensed by detecting the deflection of a reflected optical beam with a split photodetector. With low stiffness cantilevers (1 N/m), AFM instruments typically are capable of detecting forces as small as $10 \mathrm{pN}$.

Interferometric detection of cantilever deflection is also possible [17]. For cantilevers with stiffness of $1 \mathrm{~N} / \mathrm{m}$, interferometric detection allows force sensing at or below $1 \mathrm{pN}$. For ultrathin cantilevers, this capability can be extended to better than 1 aN [18].

Tunneling displacement transducers have also been explored by many researchers for sensing applications [1922]. These sensors are capable of detecting displacements of pm. Because of limitations imposed by van der waals attraction, tunneling transducers use stiffness of $1 \mathrm{~N} / \mathrm{m}$ or more to detect forces as small a $1 \mathrm{pN}$.

In summary, MEMS devices have been described which are based on a wide variety of displacement transducers. Briefly, it has been shown that piezoresistive, optical, capacitive, and tunneling transducers are all capable of force detection ranging from $\mathrm{mN}$ to $\mathrm{pN}$, and some are capable of extension to $\mathrm{fN}$ or $\mathrm{aN}$ with some effort. This very broad force measuring capability is central to the broad array of applications for MEMS devices.

\section{FORCE SENSING IN BIOLOGY}

There has been great and increasing interest among Biological scientists in the mechanical behavior of molecules, cells, and larger structures. Theoretical and experimental studies of "folding", "sticking", and "stretching" of molecules and cellular structures are widespread. Research into the fundamentals of muscle actuation, cell adhesion, protein folding, and other fundamental aspects of micro and macro Biology produces much of the most interesting contributions to Nature and Science. From our perspective, these are all questions that are very familiar to the discipline of Mechanical Engineering. In some ways, much of modern biology is beginning to look like applied mechanical engineering.

The tools that biological scientists have developed for research are not always appropriate for these applications.
Centrifuges, Microscopes, X-ray crystallography and others are not well suited to the study of the interesting structures in situ.

What is needed is a new set of custom tools for interacting with these biological systems. These tools must have the right size scale to interface to molecules, cells, or larger structures, and must have the appropriate sensitivities for extraction of interesting signals. MEMS provides a tool fabrication technology that can be adapted to many of these problems. The following list of examples shows the breadth of opportunity :

- Measurements of the binding forces of the DNA double helix show that it should be possible to distinguish the bonds and sequence DNA with resolution of $10 \mathrm{pN}$ [23]

- The forces exerted on actin filaments by single myosin "molecular motors" is between 5 and $100 \mathrm{pN}$ [24]

- The molecules responsible for cell adhesion are capable of maintaining adhesion forces of $10-100 \mathrm{pN}$ [25]

- The forces at work in the process of protein folding have been characterized energetically and shown to be in the $0.1-100 \mathrm{pN}$ range. [26]

- Individual muscles in small insects (cockroaches) have been studied as examples of efficient microactuators, and are known to exert forces in the $\mu \mathrm{N}-\mathrm{mN}$ range [27].

There are many other examples, but we easily see that the range of biological force sensing applications maps to the range of forces that can be detected by MEMS devices. In addition, it is possible to design MEMS force sensors with dimensions that are compatible with the physical geometries of these biological science applications.

\section{CELL ADHESION EXPERIMENTS}

The adhesion of individual cells is mediated by Cadherin Molecules, which extend from the actin filament structure inside of cells through the cell membrane. The ends of Cadherin molecules consist of a number of extra-cellular domains that can become attached to complimentary molecules from adjacent cells. The interactions between cadherin molecules cannot be directly detected using conventional biological science instrumentation, but it is possible to use the AFM and custom AFM cantilevers for these studies. Based on studies with whole cells, the adhesion forces for individual molecules are expected to be near $30 \mathrm{pN}$ [28]. AFM studies of molecular interactions have been carried out by many researchers $[29,30]$.

We have adapted a commercial AFM for experiments on individual cadherin molecules. The AFM must allow operation of the cantilever underwater, and the AFM cantilever and substrate must be prepared with an appropriate coating of cadherin molecules. In practice, this preparation is complicated, because it is not sufficient to simply coat the surface with cadherin molecules. In order to achieve binding that is similar to the interaction in a cellular system, it is necessary to build a surface that will cause the cadherin molecules to extend vertically. We accomplish this by coating the surface with a sequence of biotin, neutravadin, biotinalyted proteins, and then with cadherins that are initiated from a complimentary protein. This chain of support material must all feature binding strength that is greater than the adhesion forces expected from the cadherins. Experiments using fluorescent tags are carried out at each stage of the assembly to verify selective adhesion with known densities. 
In order to produce a surface with a known area that will be close enough to the opposing surface for molecular interaction, we attach known-diameter polystyrene beads to the ends of the cantilever prior to the molecular preparations.

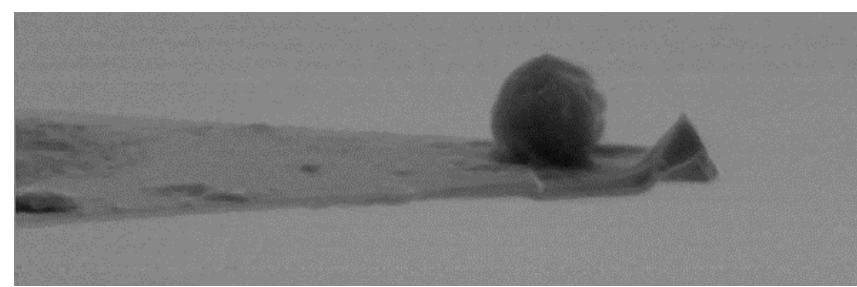

Figure 3 SEM micrograph of a $2 \mu \mathrm{m}$ diameter polystyrene bead attached to the end of a microfabricated cantilever for Cadherin Adhesion experiments.

After preparation of the cantilever and substrate, adhesion experiments are carried out under control of the AFM. The tip of the cantilever is brought into contact with the surface, and the deflection is measured as the cantilever is retracted. A typical retraction curve is shown in figure 4. Starting from the upper right, the cantilever is backed away from the surface. As the deflection is reduced, the measured force also reduces. As we pass the neutral deflection point, the cantilever tip remains attached to the surface because of the adhesive force of the Cadherin molecules. After some overshoot, some or all of the adhesion force breaks free, and the cantilever deflection is reduced. These "steps" in the cantilever retraction curve are understood to represent breaking of individual molecular adhesions. In some cases, the entire attachment is broken in a single step. In other cases, such as the example shown in Figure 4, there appears to be at least 2 distinct detachment events. In the data shown in this figure, these two events each appear to be about $70 \mathrm{pN}$ in magnitude. This experiment is repeated many times (hundreds to thousands), and histograms of attachment force are gathered to identify the values of attachment force for single molecules.

This work is in progress in our group, and we have already begun to observe variations in adhesion behavior as a function of Cadherin density, solution $\mathrm{pH}$ and calcium content, and are exploring the behavior as a function of contact force and duration. One significant advantage of this approach is that studies as a function of controllable dynamical parameters are possible, allowing a variety of interesting experiments. As a result, there is a rapidly-growing community of researchers utilizing AFM-based instruments and standard or custom cantilevers to carry out molecular biology experiments. New cantilevers with integrated heaters, multiple-axis force sensing, or other unique sensitivities will enhance this research field, and will provide continuing opportunities for MEMS researchers.

\section{INSECT BIOMECHANICS EXPERIMENTS}

Insects provide interesting examples of miniature system operation, as well as high-performance locomotion. For example, cockroaches are able to navigate complicated environments at high speed without difficulty. In contrast, the best man-made miniature robots are generally slow, and are easily trapped within complicated terrain. Sophisticated measurements of the terrain and high-level robotic maneuvering algorithms are required to function reliably. The contrast in these two examples has led researchers in "biomimetics" projects to try to learn more about cockroach biomechanics so that small man-made robots can acquire the same features.

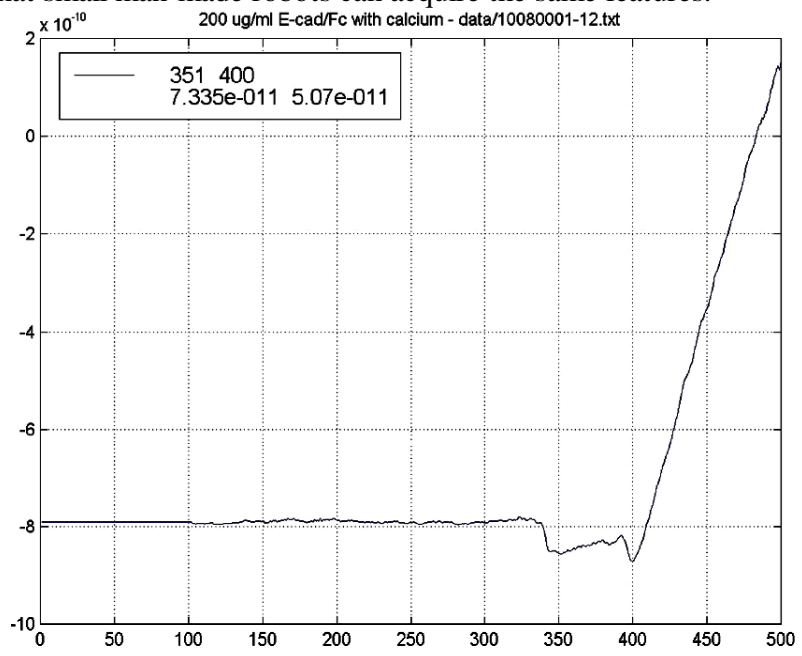

Figure 4 Plot of cantilever contact force as a function of handle displacement. In this curve, the units on the vertical axis are Hundreds of $\mathrm{pN}$, and the units on the horizontal axis are angstroms of displacement. The glitches at the lower right of the figure represent single molecule detachment events.

The first step in such a program is to carry out measurements of cockroach biomechanics. This is difficult because cockroaches are too small and too fast for macroscopic instrumentation. Load cells and force platforms cannot resolve the small forces from real cockroaches, and the dynamic response of such instruments is too slow to capture some of the more important elements of the roach behavior. Since the interesting forces can be as large as $\mathrm{mN}$, and the details are in the $\mu \mathrm{N}$ range, custom MEMS instrumentation.

Our group has been developing MEMS force platforms with integrated multiple-axis piezoresistive force sensors. Because of the relatively large forces involved (for MEMS), these structures are based on relatively thick $(40 \mu \mathrm{m})$ flexures, and required adaptation of our fabrication processes for such thick structures. SEMs of the MEMS force plate are shown in Figure 5 below. Each corner of the force plate is supported by a thin flexure with a 2-axis piezoresistive force sensor.

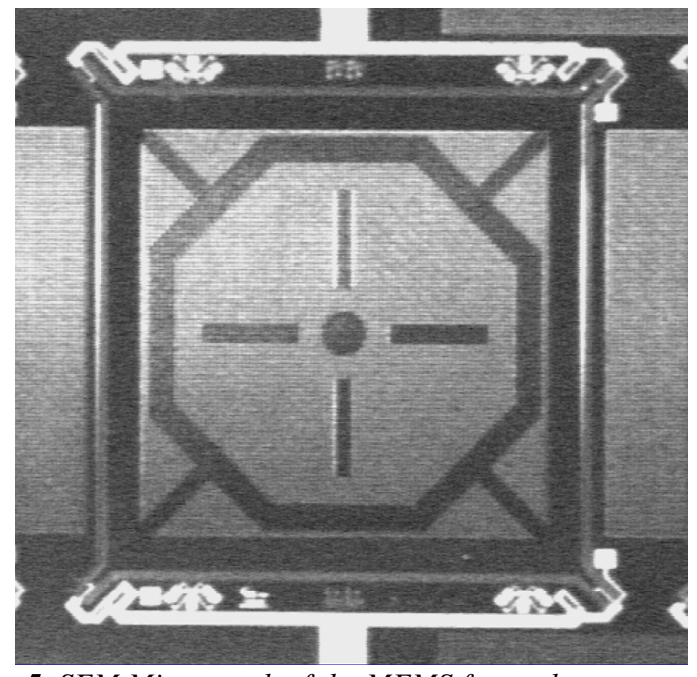

Figure 5 SEM Micrograph of the MEMS force plate 


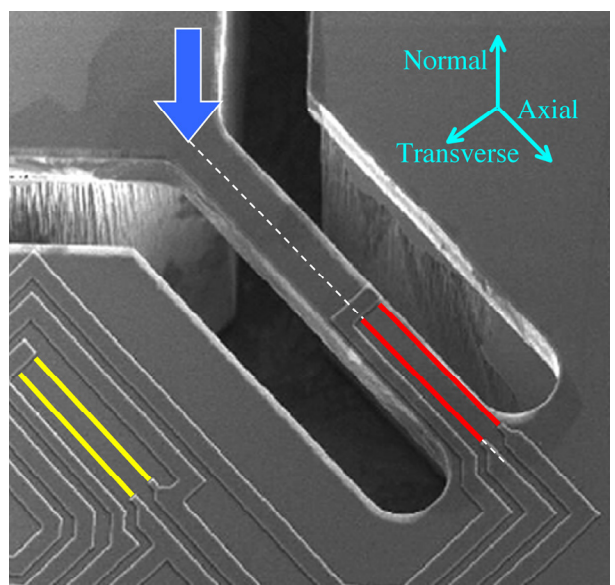

Figure 6 Close-up SEM of flexure supporting the force plate.

These microfabricated force plates are capable of resolving $10 \mathrm{nN}$ force signals in the vertical and lateral directions, and have been used for measurements on live, free-running American cockroaches and carpenter ants. These measurements are the first ever performed on cockroaches lighter than $1 \mathrm{gm}$, and the first ever performed on ants of any kind by any means. A series of representative force measurements for a running cockroach are shown in Figure 7, and for a walking ant in figure 8. In each of these measurements, there is a distinct "double -bump" in the normal force signal, which is consistent with observations on much larger animals. The two "bumps" are $r$ due to heel-strike and toe-push-off in normal walking, with the dip inbetween attributed to flexing of the "knee". From this data, we see that these insects walk in a manner that is very much like the walking of much larger creatures. Perhaps we should make robots which walk this way!

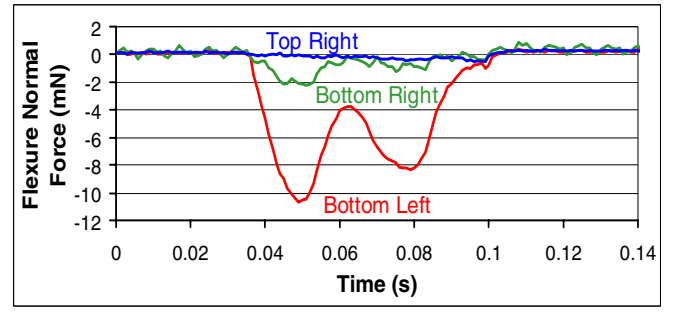

Figure 7 Time trace of the leg forces measured for an American cockroach running across the force platform.

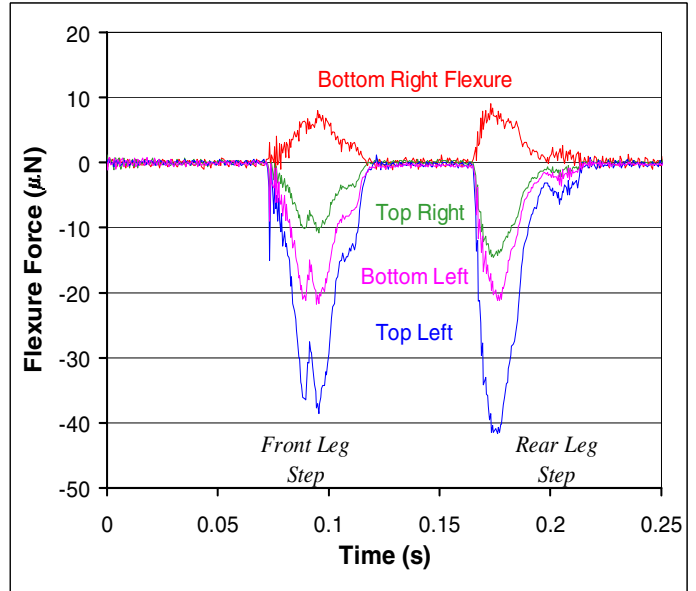

Figure 8 Time traces of the leg forces measured for a carpenter ant walking across the force platform.

\section{MEASUREMENT OF GECKO ADHESION}

Geckos are remarkable creatures, primarily for their unusual ability to cling to vertical and inverted surfaces. The adhesion mechanism has been the subject of speculation for more than 100 years [31], but definitive measurements have been difficult to carry out because of the microscopic origin of these adhesion forces. Figure 9 shows a SEM micrograph of one of the hairs on the foot of a Tokay gecko, and a close-up of the end of this hair. This hair terminates in a dense patch of sub-micron spatula that are crucial to the adhesion. Our hypothesis is that the adhesion process is due to van der waals force between the flattened ends of these spatula and the irregular shape of the opposing surface.

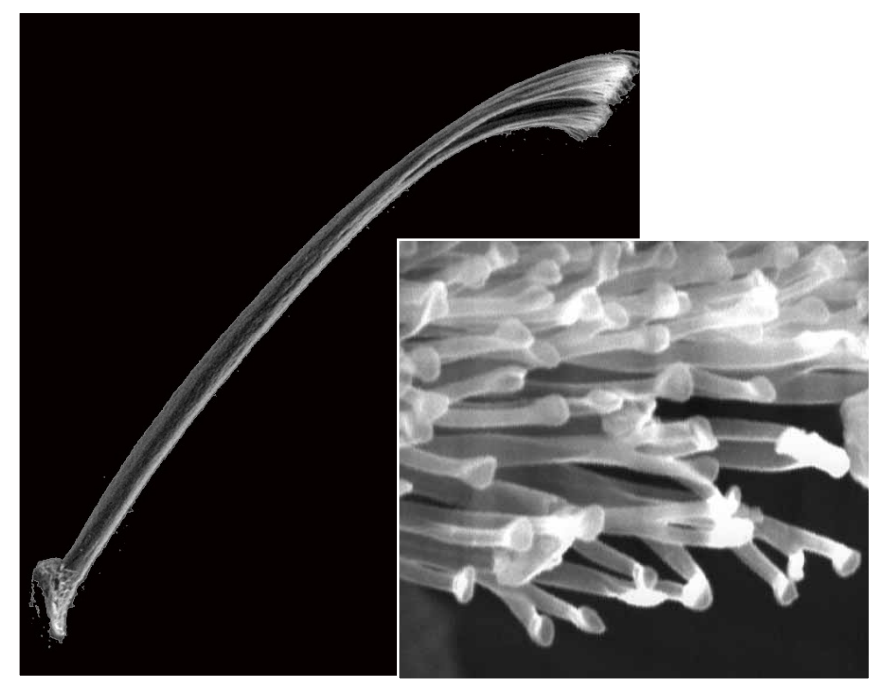

Figure 9 SEM Micrograph of a Setae from the toe of a Tokay Gecko. The microstructures on the end of the toe are shown in the inset. The flattened ends of these microstructures are less than $1 \mu \mathrm{m}$ in area, and a Tokay has more than 1 million such hairs on its feet. (SEM courtesy of Kellar Autumn)

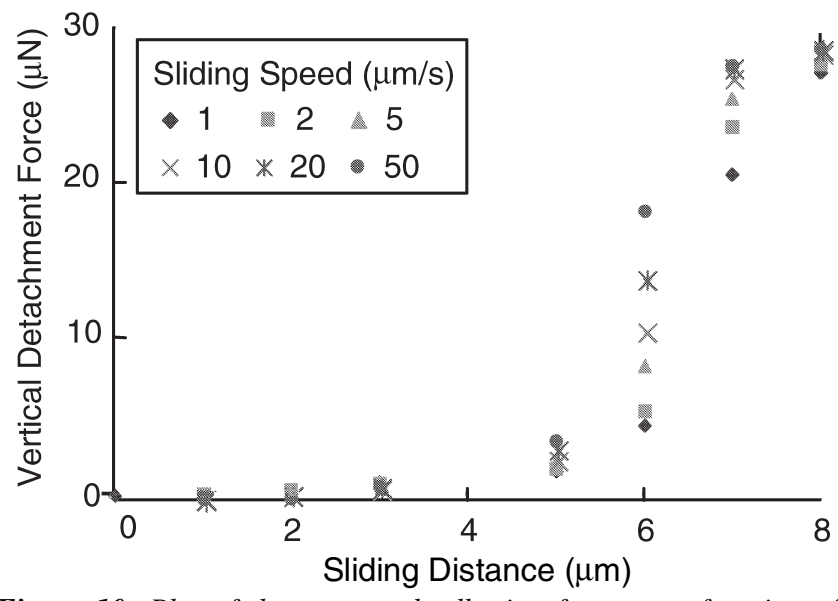

Figure 10 Plot of the measured adhesion force as a function of the lateral sliding distance recorded at a series of different sliding velocities.

At Hilton Head '00, we reported on some preliminary measurements of adhesion of individual Setae on a custom 2axis micromechanical force sensor [32], which showed that the magnitude of the adhesion force is consistent with what should be expected for van der waals force. We also saw that the adhesive force is enhanced by lateral sliding across the 
opposing surface. More recently, we have measured the amplitude of the adhesion force as a function of lateral sliding

distance and for a variety of different sliding velocities, and determined that sliding of about $5 \mu \mathrm{m}$ leads to a significant increase in adhesion, independent of the sliding speed. This data is shown in Figure 10 We believe that this result indicates that the sliding produces a kind of "combing" effect on the microstructure, and that the absence of a speed dependence shows that this effect is not related to the dynamic properties of the structure.

\section{CONCLUSIONS}

In this paper, the force sensing capabilities of MEMS devices have been discussed, and related to opportunities for measurements in biology. We've presented examples of measurements at the molecular level, at the whole-insect level, and an exploration of nano-scale adhesion mechanisms. There are many more opportunities for MEMS to contribute to biological science measurements.

\section{ACKNOWLEDGEMENTS}

The authors acknowledge collaborative support from R. Full, W. Heberle, and T. Hsieh at UC Berkeley, K. Autumn at Lewis and Clark, and J, Nelson, J. Spudich, M. Cutkosky, and their groups at Stanford. This work was supported by MURI/ONR Biomimetics Program (N-00014-98-1-0669), and by NSF XYZ on a Chip Program (CMS-9980838-002).

\section{REFERENCES}

1. K.E. Petersen, "Silicon as a Mechanical Material," Proc. IEEE, 70, 420-457 (1982).

2. Lucas Novasensor NP301, Fremont, CA.

3. J.K. Reynolds, D.C. Catling, R.C. Blue, N. Maluf, T.W. Kenny, "Packaging a Piezoresistive Pressure Sensor for Pressure and Temperature Stability in a Martian Environment," Sensors and Actuators 83, 142-149 (2000).

4. See Lucas Novasensor, ICSensors, Endevco for examples.

5. A. Partridge, et. al. "A High-Performance Planar Piezoresistive Accelerometer," JMEMS 9, 58-66 (2000).

6. F. Gretillat, M. Gretillat, and N.F. de Rooij, "Improved Design of a Silicon Micromachined Gyroscope with Piezoresistive Detection and Electromagnetic Excitation," JMEMS 8, 243 (1999).

7. M. Tortonese, "Force Sensors for Scanning Probe Microscopy," PhD Thesis, Stanford University (1993).

8. J.A. Harley, and T.W. Kenny, "High Sensitivity Piezoresistive Cantilevers under $1000 \AA$ Thick," Appl. Phys. Lett. 75, 289 (1999).

9. Y. Liang, S-W Ueng, T.W. Kenny, "Performance Characteristics of Ultra-Thin n-Type Piezoresistive Cantilevers", Proceedings Transducers '01, Munich Germany, p. 998 (2001).

10. Analog Devices ADXL202 Data Sheet, Cambridge, MA.

11. T. B. Gabrielson, "Mechanical-thermal Noise in Micromachined Acoustic and Vibration Sensors," IEEE Electron Devices, 40, 903 (1993).

12. Guralp CMG-40T, Guralp Systems Limited, Berks, UK.

13. QA3000, Honeywell Inertial Products, Redmond, WA.
14. C.H. Liu, and T.W. Kenny, A High-Precision, WideBandwidth Micromachined Tunneling Accelerometer, JMEMS, 10,425 (2001)

15. F. Ayazi and K. Najafi, "A HARPSS Polysilicon Vibrating Ring Gyroscope”, JMEMS 10, 169 (2001).

16. J.A. Geen, "A Path to Low-Cost Gyroscopy", Proceedings 1998 Hilton Head Workshop on Solid State Sensors and Actuators, 51 (1998).

17. D. Rugar, H.J. Mamin, and P. Guethner, "Improved FiberOptic Interferometer for Atomic Force Microscope," Appl. Phys. Lett. 55, 2588 (1989).

18. H.J. Mamin and D. Rugar, "Sub-Attonewton Force Detection at Millikelvin Temperatures", Appl. Phys. Lett. 79, 3358 (2001).

19. T.W. Kenny, et.al., "Micromachined Infrared Sensors using Tunneling Displacement Transducers," Rev. Sci. Instrument. 67, 112 (1996).

20. R.L. Kubena, D.J. Vickers-Kirby, R.J. Joyce, and F.P. Stratton, "A New Tunneling-based Sensor for Inertial Rotation Rate Measurements," JMEMS 8, 439 (1999).

21. C. Yeh, and K. Najafi, "Micromachined Tunneling Accelerometer with a Low-Voltage CMOS Interface Circuit," in Proceedings 1997 International Conference on Solid State Sensors and Actuators 1213 (1997).

22. J. Wang, B. McClelland, P.M. Zavracky, N.E. McGruer, and R.H. Morrison, "Study of Tunneling Noise Using SurfaceMicromachined Tunneling Tip Devices," 1997 International Conference on Solid State Sensors and Actuators 467 (1997).

23. Lee, G.U., Kidwell, D.A., Colton, R.J., "Sensing discrete streptavidin-biotin interactions with atomic force microscopy," Langmuir, 10, 354 (1994).

24. Moores, S.L., Sabry, J.H., and Spudich, J.A. "Myosin dynamics in live Dictyostelium cells (green fluorescent protein)”, Proc. Natl. Acad. Sci. 93, 443-446 (1996).

25. C.L. Adams, Y-T Chen, S.J. Smith, W.J. Nelson, "Mechanisms of Epithelial Cell-Cell Adhesion and Cell Compaction Revealed by High-resolution Tracking of ECadherin-Green Fluorescent Protein," The Journal of Cell Biology 142, 1105 (1998).

26. M. Rief et.al. "Reversible unfolding of individual titin immunoglobulin domains by AFM," Science 276 (5315) : 1109 (1997).

27. K. Autumn, et.al., "How Gecko Feet Work", American Zoologist 39, 621 (1999).

28.A.S. Yap, W.M. Brieher, M. Pruschy, B.M. Gumbiner, "Lateral clustering of the adhesive ectodomain: A fundamental determinant of cadherin function", Current Biology 7, 308 (1997)

29. T. Hugel, et.al., "Elasticity of single polyelectrolyte chains and their desorption from solid supports studied by AFM based single molecule force spectroscopy”, Macromolecules 34, 1039 (2001)

30. W. Baumgartner, et.al., "Determination of the unbinding force of hemophilic interaction of vascular endothelial cadherin by atomic force microscopy", Biophysics Journal 76, A351 (1999).

31. H.R. Schmidt, "Zur Anatoomie und Physiologie der Geckopfote”, Jena Z. Naturw 39, 551 (1904).

32. K. Autumn, Y. Liang, W.P. Chan, T. Hsieh, R. Fearing, T.W. Kenny, and R. Full, Dry Adhesive Force of a Single Gecko Foot-Hair, Nature. 405: 681-685 (2000). 